\title{
Perspectives for Higgs and New Physics
}

\author{
Abdelhak Djouadi ${ }^{1, a}$ \\ ${ }^{1}$ Laboratoire de Physique Théorique, CNRS \& Université Paris-Sud, 91405 Orsay, France
}

\begin{abstract}
The implications of the discovery of a Higgs boson at the LHC with a mass of $125 \mathrm{GeV}$ are summarised in the context of the Standard Model of particle physics and in new physics scenarios beyond it, taking the example of the minimal supersymmetric Standard Model extension, the MSSM. The perspectives for Higgs and new physics searches at the next LHC upgrades as well as at future hadron and lepton colliders are then briefly summarized.
\end{abstract}

\section{Introduction}

The ATLAS and CMS historical discovery of a particle with a mass of $125 \mathrm{GeV}$ [1] and properties that are compatible with those of a scalar Higgs boson [2,3] has far reaching consequences not only for the Standard Model (SM) but also for new physics models beyond it. In the SM, electroweak symmetry breaking is achieved spontaneously via the Brout-Englert-Higgs mechanism [2], wherein the neutral component of an isodoublet scalar field acquires a non-zero vacuum expectation value $v$. This gives rise to nonzero masses for the fermions and the electroweak gauge bosons while preserving the $\mathrm{SU}(2) \times \mathrm{U}(1)$ gauge symmetry. One of the four degrees of freedom of the original isodoublet field, corresponds to a physical particle [3]: a scalar boson with $\mathrm{J}^{\mathrm{PC}}=0^{++}$quantum numbers under parity and charge conjugation. The couplings of the Higgs boson to the fermions and gauge bosons are related to the masses of these particles and are thus decided by the symmetry breaking mechanism. In contrast, the Higgs mass itself $M_{H}$, although expected to be in the vicinity of the weak scale $v \approx 250$ $\mathrm{GeV}$, is undetermined. Let us summarise the known information on this parameter before the start of the LHC.

A direct information was the lower limit $M_{H} \gtrsim 114 \mathrm{GeV}$ at $95 \%$ confidence level (CL) established at LEP2 [4]. Furthermore, a global fit of the electroweak precision data to which the Higgs boson contributes, yields the value $M_{H}=92_{-26}^{+34} \mathrm{GeV}$, corresponding to a 95\% CL upper limit of $M_{H} \lesssim 160$ $\mathrm{GeV}$ [4]. From the theoretical side, the presence of this new weakly coupled degree of freedom is a crucial ingredient for a unitary electroweak theory. Indeed, the SM without the Higgs particle leads to scattering amplitudes of the $W / Z$ bosons that grow with the square of the center of mass energy and perturbative unitarity would be lost at energies above the $\mathrm{TeV}$ scale. In fact, even in the presence of a Higgs boson, the $W / Z$ bosons could interact very strongly with each other and, imposing the unitarity requirement leads to the important mass bound $M_{H} \lesssim 700 \mathrm{GeV}$ [5], implying that the particle is kinematically accessible at the LHC.

ae-mail: abdelhak.djouadi@cern.ch 
Another theoretical constraint emerges from the fact that the Higgs self-coupling, $\lambda \propto M_{H}^{2}$, evolves with energy and at some stage, becomes very large and even infinite and the theory completely looses its predictability. If the energy scale up to which the couplings remains finite is of the order of $M_{H}$ itself, one should have $M_{H} \lesssim 650 \mathrm{GeV}$ [6]. On the other hand, for small values of $\lambda$ and hence $M_{H}$, the quantum corrections tend to drive the self-coupling to negative values and completely destabilize the scalar Higgs potential to the point where the minimum is not stable anymore [6]. Requiring $\lambda \geq 0$, up to the $\mathrm{TeV}$ scale implies that $M_{H} \gtrsim 70 \mathrm{GeV}$. If the $\mathrm{SM}$ is to be extended to the Planck scale $\mathrm{M}_{\mathrm{P}} \sim 10^{18} \mathrm{GeV}$, the requirements on $\lambda$ from finiteness and positivity constrain the Higgs mass to lie in the range $130 \mathrm{GeV} \lesssim M_{H} \lesssim 180 \mathrm{GeV}$ [6]. This narrow margin is close to the one obtained from the direct and indirect experimental constraints.

The discovery of the Higgs particle with a mass of $125 \mathrm{GeV}$, a value that makes the SM perturbative, unitary and extrapolable to the highest possible scales, is therefore a consecration of the model and crowns its past success in describing all experimental data available. In particular, the average mass value measured by the ATLAS and CMS teams, $M_{H}=125.1 \pm 0.24 \mathrm{GeV}$ [7], is remarkably close to the best-fit of the precision data which should be considered as a great achievement and a triumph for the SM. In addition, a recent analysis that includes the state-of-the-art quantum corrections [8] gives for the condition of absolute stability of the electroweak vacuum, $\lambda\left(M_{P}\right) \geq 0$, the bound $M_{H} \gtrsim 129 \mathrm{GeV}$ for the present value of the top quark mass and the strong coupling constant, $m_{t}^{\exp }=173.2 \pm 0.9 \mathrm{GeV}$ and $\alpha_{s}\left(M_{Z}\right)=0.1184 \pm 0.0007$ [4]. Allowing for a $2 \sigma$ variation of $m_{t}^{\exp }$, one obtains $M_{H} \geq 125.6 \mathrm{GeV}$ that is close to the measured $M_{H}$ value [7]. In fact, for an unambiguous and well-defined determination of the top mass, one should rather use the total cross section for top pair production at hadron colliders which can unambiguously be defined theoretically; this mass has a larger uncertainty, $\Delta m_{t} \approx 3 \mathrm{GeV}$, which allows more easily absolute stability of the SM vacuum up to $M_{P}$ [9].

Nevertheless, the SM is far from being perfect in many respects. It does not explain the proliferation of fermions and the large hierarchy in their mass spectra and does not say much about the small neutrino masses. The SM does not unify in a satisfactory way the electromagnetic, weak and strong forces, as one has three different symmetry groups with three coupling constants which shortly fail to meet at a common value during their evolution with the energy scale; it also ignores the fourth force, gravitation. Furthermore, it does not contain a particle that could account for the cosmological dark matter and fails to explain the baryon asymmetry in the Universe.

However, the main problem that calls for beyond the SM is related to the special status of the Higgs boson which, contrary to fermions and gauge bosons has a mass that cannot be protected against quantum corrections. Indeed, these are quadratic in the new physics scale which serves as a cut-off and hence, tend to drive $M_{H}$ to very large values, ultimately to $M_{P}$, while we need $M_{H}=O(100 \mathrm{GeV})$. Thus, the SM cannot be extrapolated beyond $O(1 \mathrm{TeV})$ where some new physics should emerge. This is the reason why we expect something new to manifest itself at the LHC.

There are three avenues for the many new physics scenarios beyond the SM. There are first theories with extra space-time dimensions that emerge at the $\mathrm{TeV}$ scale (the cut-off is then not so high) and, second, composite models inspired from strong interactions also at the TeV scale (and thus the Higgs is not a fundamental spin-zero particle). Some versions of these scenarios do not incorporate any Higgs particle in their spectrum and are thus ruled out by the Higgs discovery. However, the option that emerges in the most natural way is Supersymmetry (SUSY) [10] as it solves most of the SM problems discussed above. In particular, SUSY protects $M_{H}$ as the quadratically divergent radiative corrections from standard particles are exactly compensated by the contributions of their supersymmetric partners. These new particles should not be much heavier than $1 \mathrm{TeV}$ not to spoil this compensation [11] and, thus, they should be produced at the LHC. 
The Higgs discovery is very important for SUSY and, in particular, for its simplest low energy manifestation, the minimal supersymmetric SM (MSSM) that indeed predicts a light Higgs state. In the MSSM, two Higgs doublet fields $H_{u}$ and $H_{d}$ are required, leading to an extended Higgs consisting of five Higgs bosons, two CP-even $h$ and $H$, a CP-odd $A$ and two charged $H^{ \pm}$states [12]. Nevertheless, only two parameters are needed to describe the Higgs sector at tree-level: one Higgs mass, which is generally taken to be that of the pseudoscalar boson $M_{A}$, and the ratio of vacuum expectation values of the two Higgs fields, $\tan \beta=v_{d} / v_{u}$, expected to lie in the range $1 \lesssim \tan \beta \lesssim 60$. The masses of the $\mathrm{CP}$-even $h, H$ and the charged $H^{ \pm}$states, as well as the mixing angle $\alpha$ in the $\mathrm{CP}$-even sector are uniquely defined in terms of these two inputs at tree-level, but this nice property is spoiled at higher orders [13]. For $M_{A} \gg M_{Z}$, one is in the so-called decoupling regime in which the $h$ state is light and has almost exactly the SM-Higgs couplings, while the other $\mathrm{CP}$-even $H$ and the charged $H^{ \pm}$bosons become heavy, $M_{H} \approx M_{H^{ \pm}} \approx M_{A}$, and decouple from the massive gauge bosons. In this regime, the MSSM Higgs sector thus looks almost exactly as the one of the SM with its unique Higgs boson.

Nevertheless, contrary to the SM Higgs boson, the lightest MSSM CP-even $h$ mass is bounded from above and, depending on the SUSY parameters that enter the important quantum corrections, is restricted to $M_{h}^{\max } \lesssim 130 \mathrm{GeV}$ [13] if one assumes a SUSY breaking scale that is not too high, $M_{S} \lesssim O(1 \mathrm{TeV})$, in order to avoid too much fine-tuning in the model. Hence, the requirement that the MSSM $h$ boson coincides with the one observed at the LHC, i.e. with $M_{h} \approx 125 \mathrm{GeV}$ and almost SMlike couplings as the LHC data seem to indicate, would place very strong constraints on the MSSM parameters, in particular the SUSY-breaking scale $M_{S}$. This comes in addition to the LHC limits obtained from the search of the heavier Higgs states and the superparticles.

In this talk, the implications of the discovery of the Higgs boson at the LHC and the measurement of its properties will be summarised and the prospects for the searches of new physics, in particular in the SUSY context, in the future will be summarized.

\section{Implications for the Standard Model and Supersymmetry}

In many respects, the Higgs particle was born under a very lucky star as the mass value of $\approx 125$ $\mathrm{GeV}$ allows to produce it at the LHC in many redundant channels and to detect it in a variety of decay modes. This allows detailed studies of the Higgs properties.

\subsection{Higgs production and decay}

We start by summarizing the production and decay at the LHC of a light SM-like Higgs particle, which should correspond to the lightest MSSM $h$ boson in the decoupling regime. First, for $M_{H} \approx 125$ $\mathrm{GeV}$, the Higgs mainly decays [14] into $b \bar{b}$ pairs but the decays into $W W^{*}$ and $Z Z^{*}$ final states, before allowing the gauge bosons to decay leptonically $W \rightarrow \ell v$ and $Z \rightarrow \ell \ell(\ell=\ell, \mu)$, are also significant. The $H \rightarrow \tau^{+} \tau^{-}$channel (as well as the $g g$ and $c \bar{c}$ decays that are not detectable at the LHC) is also of significance, while the clean loop induced $H \rightarrow \gamma \gamma$ mode can be easily detected albeit its small rates. The very rare $H \rightarrow Z \gamma$ and even $H \rightarrow \mu^{+} \mu^{-}$channels should be accessible at the LHC but only with a much larger data sample.

On the other hand, many Higgs production processes have significant cross sections [15-17]. While the by far dominant gluon fusion mechanism $g g \rightarrow H(\mathrm{ggF})$ has extremely large rates $(\approx 20 \mathrm{pb}$ at $\sqrt{s}=7-8 \mathrm{TeV}$ ), the subleading channels, i.e. the vector boson fusion (VBF) $q q \rightarrow H q q$ and the Higgs-strahlung (HV) $q \bar{q} \rightarrow H V$ with $V=W, Z$ mechanisms, have cross sections which should allow for a study of the Higgs particle already at $\sqrt{s} \gtrsim 7 \mathrm{TeV}$ with the $\approx 25 \mathrm{fb}^{-1}$ data collected by each experiment. The associated process $p p \rightarrow t \bar{t} H(\mathrm{ttH})$ would require higher energy and luminosity.

This pattern already allows the ATLAS and CMS experiments to observe the Higgs boson in several channels and to measure some of its couplings in a reasonably accurate way. The channels 
that have been searched are $H \rightarrow Z Z^{*} \rightarrow 4 \ell^{ \pm}, H \rightarrow W W^{*} \rightarrow 2 \ell 2 v, H \rightarrow \gamma \gamma$ where the Higgs is mainly produced in ggF with subleading contributions from $H j j$ in the VBF process, $H \rightarrow \tau \tau$ where the Higgs is produced in association with one (in ggF) and two (in VBF) jets, and finally $H \rightarrow b \bar{b}$ with the Higgs produced in the $\mathrm{HV}$ process. One can ignore for the moment the low sensitivity $H \rightarrow \mu \mu$ and $H \rightarrow Z \gamma$ channels.

A convenient way to scrutinize the couplings of the produced $H$ boson is to look at their deviation from the SM expectation. One then considers for a given search channel the signal strength modifier $\mu$ which for the $H \rightarrow X X$ decay mode measures the deviation compared to the SM expectation of the Higgs production cross section times decay branching fraction $\mu_{X X}$. ATLAS and CMS have provided the signal strengths for the various final states with a luminosity of $\approx 5 \mathrm{fb}^{-1}$ for the 2011 run at $\sqrt{s}=7$ $\mathrm{TeV}$ and $\approx 20 \mathrm{fb}^{-1}$ for the 2012 run at $\sqrt{s}=8 \mathrm{TeV}$. The constraints given by the collaborations, when combined, lead to a global signal strength $\mu_{\text {tot }}^{\text {ATLAS }}=1.18 \pm 0.15$ and $\mu_{\text {tot }}^{\mathrm{CMS}}=1.00 \pm 0.14$ [7]. The global value being very close to unity implies that the observed Higgs is SM-like. Hence, already with the rather limited statistics at hand, the accuracy of the ATLAS and CMS measurements is reaching the $15 \%$ level.

This is at the same time impressive and worrisome. Indeed, the main Higgs production channel is the top and bottom quark loop mediated gluon fusion mechanism and, at $\sqrt{s}=7$ or $8 \mathrm{TeV}$, the three other mechanisms contribute at a total level below $15 \%$. The majority of the signal events observed at LHC, in particular in the search channels $H \rightarrow \gamma \gamma, H \rightarrow Z Z^{*} \rightarrow 4 \ell, H \rightarrow W W^{*} \rightarrow 2 \ell 2 v$ and to some extent $H \rightarrow \tau \tau$, thus come from the $\mathrm{ggF}$ mechanism which is known to be affected by large theoretical uncertainties.

Indeed, although $\sigma(g g \rightarrow H)$ is known up next-to-next-to-leading order (NNLO) in perturbative QCD (and at least at NLO for the electroweak interaction) $[15,16]$, there is a significant residual scale dependence which points to the possibility that still higher order contributions cannot be totally excluded. In addition, as the process is of $O\left(\alpha_{s}^{2}\right)$ at LO and is initiated by gluons, there are sizable uncertainties due to the gluon parton distribution function (PDF) and the value of the coupling $\alpha_{s}$. A third source of theoretical uncertainties, the use of an effective field theory (EFT) approach to calculate the radiative corrections beyond NLO should also be considered [15]. In addition, large uncertainties arise when $\sigma(g g \rightarrow H)$ is broken into the jet categories $H+0 j, H+1 j$ and $H+2 j$ [18]. In total, the combined theoretical uncertainty is estimated to be $\Delta^{\text {th }} \approx \pm 15 \%$ [16] and would increase to $\Delta^{\text {th }} \approx \pm 20 \%$ if the EFT uncertainty is also included. The a priori cleaner VBF process will be contaminated by the $g g \rightarrow H+2 j$ mode making the total uncertainty in the $H+j j$ "VBF" sample also rather large [18].

Hence, the theoretical uncertainty is already at the level of the accuracy of the cross section measured by the ATLAS and CMS collaborations. Another drawback of the analyses is that they involve strong theoretical assumptions on the total Higgs width since some contributing decay channels not accessible at the LHC are assumed to be SM-like and possible invisible Higgs decays in scenarios beyond the SM do not to occur.

In Ref. [17], following earlier work [19] it has been suggested to consider the ratio $D_{X X}^{\mathrm{p}}=\sigma^{\mathrm{p}}(p p \rightarrow$ $H \rightarrow X X) / \sigma^{\mathrm{p}}(p p \rightarrow H \rightarrow V V)$ for a specific production process $p$ and for a given decay channel $H \rightarrow X X$ when the reference channel $H \rightarrow V V$ is used. In these ratios, the cross sections and hence, their significant theoretical uncertainties will cancel out, leaving out only the ratio of partial decay widths which are better known. The total decay width which includes contributions from channels not under control such as possible invisible Higgs decays, do not appear in the ratios $D_{X X}^{\mathrm{p}}$. Some common experimental systematical uncertainties such as the one from the luminosity measurement and the small uncertainties in the Higgs decay branching ratios also cancel out. We are thus left with only with the statistical and some (non common) systematical errors [17]. 
The ratios $D_{X X}$ involve, up to kinematical factors and known radiative corrections, only the ratios $\left|c_{X}\right|^{2} /\left|c_{V}\right|^{2}$ of the Higgs reduced couplings to the particles $X$ and $V$ compared to the SM expectation, $c_{X} \equiv g_{H X X} / g_{H X X}^{\mathrm{SM}}$. For the time being, three independent ratios can be considered: $D_{\gamma \gamma}, D_{\tau \tau}$ and $D_{b b}$. In order to determine these ratios, the theoretical uncertainties have to be treated as a bias (and not as if they were associated with a statistical distribution) and the fit has to be performed for the two $\mu$ extremal values: $\left.\mu_{i}\right|_{\exp } \pm \delta \mu_{i} /\left.\mu_{i}\right|_{\text {th }}$ with $\delta \mu_{i} /\left.\mu_{i}\right|_{\text {th }} \approx \pm 20 \%$ [20].

A large number of analyses of the Higgs couplings from the LHC data have been performed and in most cases, it is assumed that the couplings of the Higgs boson to the massive $W, Z$ gauge bosons are equal to $g_{H Z Z}=g_{H W W}=c_{V}$ and the couplings to all fermions are also the same $g_{H f f}=c_{f}$. However, as for instance advocated in Ref. [21] to characterize the Higgs particle at the LHC, at least three independent $H$ couplings should be considered, namely $c_{t}, c_{b}$ and $c_{V}$. While the couplings to $W, Z, b, \tau$ particles are derived by considering the decays of the Higgs boson to these particles, the $H t \bar{t}$ coupling is derived indirectly from $\sigma(g g \rightarrow H)$ and $\mathrm{BR}(H \rightarrow \gamma \gamma)$, two processes that are generated by triangular loops involving the top quarks in the SM. One can assume, in a first approximation, that $c_{c}=c_{t}$ and $c_{\tau}=c_{b}$ and possible invisible Higgs decays are absent. In Ref. [21], a three-dimensional fit of the $H$ couplings was performed in the space $\left[c_{t}, c_{b}, c_{V}\right]$, when the theory uncertainty is taken as a bias and not as a nuisance. The best-fit value for the couplings, with the $\sqrt{s}=7+8 \mathrm{TeV}$ ATLAS and CMS data turns out to be $c_{t}=0.89, c_{b}=1.01$ and $c_{V}=1.02$, ie very close to the SM values.

\subsection{Implications of the Higgs couplings measurement in the SM}

The precise measurements of the Higgs couplings allow to draw several important conclusions.

i) A fourth generation fermions is excluded. Indeed, in addition to the direct LHC searches that exclude heavier quarks $m_{b^{\prime}}, m_{t^{\prime}} \lesssim 600 \mathrm{GeV}$ [23], strong constraints can be also obtained from the loop induced Higgs-gluon and Higgs-photon vertices in which any heavy particle coupling to the Higgs proportionally to its mass will contribute. For instance the additional 4 th generation $t^{\prime}$ and $b^{\prime}$ contributions increase $\sigma(g g \rightarrow H)$ by a factor of $\approx 9$ at LO but large $O\left(G_{F} m_{f^{\prime}}^{2}\right)$ electroweak corrections should be considered. It has been shown [23] that with a fourth family, the Higgs signal would have not been observable and the obtained Higgs results unambiguously rule out this possibility.

ii) The invisible Higgs decay width should be small. Invisible decays would affect the properties of the observed Higgs boson and could be constrained if the total decay width is determined. But for a $125 \mathrm{GeV}$ Higgs, $\Gamma_{H}^{\text {tot }}=4 \mathrm{MeV}$, is too small to be resolved experimentally. Nevertheless, in $p p \rightarrow V V \rightarrow 4 f$, a large fraction of the Higgs cross section lies in the high-mass tail [24] allowing to to put loose constrains $\Gamma_{H}^{\text {tot }} / \Gamma_{H}^{\mathrm{SM}} \approx 5-10$ [25]. The invisible Higgs decay width $\Gamma_{H}^{\text {inv }}$ can be better constrained indirectly by a fit of the Higgs couplings and in particular with the signal strength in the $H \rightarrow Z Z$ process: $\mu_{Z Z} \propto \Gamma(H \rightarrow Z Z) / \Gamma_{H}^{\text {tot }}$ with $\Gamma_{H}^{\text {tot }}=\Gamma_{H}^{\text {inv }}+\Gamma_{H}^{\mathrm{SM}}$; one obtains $\Gamma_{H}^{\text {inv }} / \Gamma_{H}^{\mathrm{SM}} \lesssim 50 \%$ at the 95\% CL if the assumption $c_{f}=c_{V}=1$ is made [20].

A more model independent approach would be to perform direct searches for missing transverse energy. These have been conducted in $p p \rightarrow H V$ with $V \rightarrow j j, \ell \ell$ and in $\mathrm{VBF}, q q \rightarrow q q E_{T}$ leading to $\mathrm{BR}_{\text {inv }} \lesssim 50 \%$ at $95 \% \mathrm{CL}$ for SM-like Higgs couplings [7]. A more promising search for invisible decays is the monojet channel $g g \rightarrow H j$ which has large rates [26]. While the most recent monojet ATLAS and CMS searches are only sensitive to $\mathrm{BR}_{\mathrm{inv}} \sim 1$, more restrictive results can be obtained in the future.

The Higgs invisible rate and the dark matter detection rate in direct astrophysical searches are correlated in Higgs portal models and it turns out that LHC constraints are competitive [27] with those derived from direct dark matter search experiments [28].

iii) The spin-parity quantum numbers are those of a standard Higgs. One also needs to establish that the observed Higgs state is indeed a CP even scalar and hence with $\mathrm{J}^{\mathrm{PC}}=0^{++}$quantum numbers. 
For the spin, the observation of the $H \rightarrow \gamma \gamma$ decay rules out the spin-1 case [29]. The Higgs parity can be probed by studying kinematical distributions in the $H \rightarrow Z Z^{*} \rightarrow 4 \ell$ decay channel and in the VH and VBF production modes [30] and with the $25 \mathrm{fb}^{-1}$ data collected so far, ATLAS and CMS found that the observed Higgs is more compatible with a $0^{+}$state and the $0^{-}$possibility is excluded at the 98\%CL [7]. Other useful diagnostics of the Higgs CP nature that also rely on the tensorial structure of the $H V V$ coupling can be made in the VBF process [31]. Nevertheless, there is a caveat in the analyses relying on the $H V V$ couplings: a $\mathrm{CP}-$ odd state has no tree-level $V V$ couplings [32]. In fact, a better way to measure the Higgs parity is to study the signal strength in the $H \rightarrow V V$ channels and in Ref. [20] it was demonstrated that the observed Higgs has indeed a large CP component, $\gtrsim 50 \%$ at the $95 \% \mathrm{CL}$. In fact, the less unambiguous way to probe the Higgs CP nature would be to look at final states in which the particle decays hadronically, e.g. $p p \rightarrow H Z \rightarrow b \bar{b} \ell \ell$ [32]. These processes are nevertheless extremely challenging even at the upgraded LHC.

\subsection{Implications for Supersymmetry}

We turn now to the implications of the LHC Higgs results for the MSSM Higgs sector and first make a remark on the Higgs masses and couplings, which at tree-level depend only on $M_{A}$ and $\tan \beta$, when the important radiative corrections are included. In this case many parameters such as the masses of the third generation squarks $m_{\tilde{t}_{i}}, m_{\tilde{b}_{i}}$ and their trilinear couplings $A_{t}, A_{b}$ enter $M_{h}$ and $M_{H}$ through quantum corrections. These are introduced by a general $2 \times 2$ matrix $\Delta \mathcal{M}_{i j}^{2}$ but the leading one is controlled by the top Yukawa coupling and is proportional to $m_{t}^{4}, \log M_{S}$ with $M_{S}=\sqrt{m_{\tilde{t}_{1}} m_{\tilde{t}_{2}}}$ the SUSY-breaking scale and the stop mixing parameter $X_{t}$ [13]. The maximal value $M_{h}^{\max }$ is then obtained for a decoupling regime $M_{A} \sim O(\mathrm{TeV})$, large $\tan \beta$, large $M_{S}$ that implies heavy stops and maximal mixing $X_{t}=\sqrt{6} M_{S}$ [33]. If the parameters are optimized as above, the maximal $M_{h}$ value reaches the level of $130 \mathrm{GeV}$.

It was pointed out in Refs. [21, 34, 35] that when the measured value $M_{h}=125 \mathrm{GeV}$ is taken into account, the MSSM Higgs sector with only the largely dominant correction discussed above, can be again described with only the two parameters $\tan \beta$ and $M_{A}$; in other words, the loop corrections are fixed by the value of $M_{h}$. This observation leads to a rather simple but accurate parametrisation of the MSSM Higgs sector, called $h$ MSSM.

The reduced couplings of the $\mathrm{CP}$-even $h$ state (as is the case for the heavier $H$ ) depend in principle only on the angles $\beta$ and $\alpha$ (and hence $\tan \beta$ and $M_{A}$ ), $c_{V}^{0}=\sin (\beta-\alpha), c_{t}^{0}=\cos \alpha / \sin \beta, c_{b}^{0}=-\sin \alpha / \cos \beta$, while the couplings of $A$ and $H^{ \pm}$(as well as $H$ in the decoupling regime) to gauge boson are zero and those to fermions depend only on $\beta$ : for $\tan \beta>1$, they are enhanced $(\alpha \tan \beta)$ for $b, \tau$ and suppressed $(\propto 1 / \tan \beta)$ for tops.

i) Implications from the Higgs mass value: In the so-called "phenomenological MSSM" (pMSSM) [37] in which the model involves only 22 free parameters, a large scan has been performed [36] using the RGE program Suspect [38] that calculates the maximal $M_{h}$ value and the result confronted to the measured mass $M_{h} \sim 125 \mathrm{GeV}$. For $M_{S} \lesssim 1 \mathrm{TeV}$, only scenarios with $X_{t} / M_{S}$ values close to maximal mixing $X_{t} / M_{S} \approx \sqrt{6}$ survive. The no-mixing scenario $X_{t} \approx 0$ is ruled out for $M_{S} \lesssim 3 \mathrm{TeV}$, while the typical mixing scenario, $X_{t} \approx M_{S}$, needs large $M_{S}$ and moderate to large $\tan \beta$ values. In constrained MSSM scenarios (cMSSM) such the minimal supergravity (mSUGRA) model and the gauge and anomaly mediated SUSY-breaking scenarios, GMSB and AMSB, only a few basic inputs are needed and the mixing parameter cannot take arbitrary values. A scan in these models with $M_{S} \lesssim 3 \mathrm{TeV}$ not to allow for too much fine-tuning [11] leads $M_{h}^{\max } \lesssim 122 \mathrm{GeV}$ in AMSB and GMSB thus disfavoring these scenarios while one has $M_{h}^{\max }=128 \mathrm{GeV}$ in mSUGRA. In high-scale SUSY scenarios, $M_{S} \gg M_{Z}$, the radiative corrections are very large and need to be resumed [39]. For low 
$\tan \beta$ values, large scales, at least $M_{S} \gtrsim 10^{4} \mathrm{GeV}$, are required to obtain $M_{h}=125 \mathrm{GeV}$ and even higher in most cases

ii) Implications from the production rates of the observed state. Besides the corrections to the Higgs masses and couplings discussed above, there are also direct corrections to the Higgs couplings and the most ones are those affecting the $h b \bar{b}$ vertex [40] and the stop loop contributions to the $g g \rightarrow h$ production and $h \rightarrow \gamma \gamma$ decay rates [41]. A fit of the $c_{t}, c_{b}$ and $c_{V}$ couplings shows that the latter are small [20]. In turn, ignoring the direct corrections and using the input $M_{h} \approx 125 \mathrm{GeV}$, one can make a fit in the plane $\left[\tan \beta, M_{A}\right]$. The best-fit point is $\tan \beta=1$ and $M_{A}=550 \mathrm{GeV}$ which implies a large SUSY scale, $M_{S}=O(100) \mathrm{TeV}$. In all, cases one also has $M_{A} \gtrsim 200-350 \mathrm{GeV}$.

iii) Implications from heavy Higgs boson searches. At high $\tan \beta$ values, the strong enhancement of the $b, \tau$ couplings makes that the $\Phi=H / A$ states decay dominantly into $\tau^{+} \tau^{-}$and $b \bar{b}$ pairs and are mainly produced in $g g \rightarrow \Phi$ fusion with the $b$-loop included and associated production with $b$-quarks, $g g / q \bar{q} \rightarrow b \bar{b}+\Phi$ [42]. The most powerful LHC search channel is thus $p p \rightarrow g g+b \bar{b} \rightarrow \Phi \rightarrow \tau^{+} \tau^{-}$. For the charged Higgs, the dominant mode is $H^{ \pm} \rightarrow \tau v$ with the $H^{ \pm}$light enough to be produced in top decays $t \rightarrow H^{+} b \rightarrow \tau v b$. In the low $\tan \beta$ regime, $\tan \beta \lesssim 3$, the phenomenology of the $A, H, H^{ \pm}$ states is richer [34]. For the production, only $g g \rightarrow \Phi$ process with the dominant $t$ and sub-dominant $b$ contributions provides large rates. The $H / A / H^{ \pm}$decay pattern is in turn rather involved. Above the $t \bar{t}(t b)$ threshold $H / A \rightarrow t \bar{t}$ and $H^{+} \rightarrow t \bar{b}$ are by far dominant. Below threshold, the $H \rightarrow W W, Z Z$ decays are significant. For $2 M_{h} \lesssim M_{H} \lesssim 2 m_{t}\left(M_{A} \gtrsim M_{h}+M_{Z}\right), H \rightarrow h h(A \rightarrow h Z)$ is the dominant $H(A)$ decay mode. But the $A \rightarrow \tau \tau$ channel is still important with rates $\gtrsim 5 \%$. In the case of $H^{ \pm}$, the channel $H^{+} \rightarrow W h$ is important for $M_{H^{ \pm}} \lesssim 250 \mathrm{GeV}$, similarly to the $A \rightarrow h Z$ case.

In Ref. [34] an analysis of these channels has been performed using current information given by ATLAS and CMS in the context of the SM, MSSM [43] or other scenarios. The outcome is impressive. The ATLAS and CMS $H / A \rightarrow \tau^{+} \tau^{-}$constraint is extremely restrictive and $M_{A} \lesssim 250 \mathrm{GeV}$, it excludes almost the entire intermediate and high $\tan \beta$ regimes. The constraint is less effective for a heavier $A$ but even for $M_{A} \approx 400 \mathrm{GeV}$ the high $\tan \beta \gtrsim 10$ region is excluded and one is even sensitive to $M_{A} \approx 800 \mathrm{GeV}$ for $\tan \beta \gtrsim 50$. For $H^{ \pm}$, almost the entire $M_{H^{ \pm}} \lesssim 160 \mathrm{GeV}$ region is excluded by the process $t \rightarrow H^{+} b$ with the decay $H^{+} \rightarrow \tau v$. The other channels, in particular $H \rightarrow V V$ and $H / A \rightarrow t \bar{t}$, are very constraining as they cover the entire low $\tan \beta$ area that was previously excluded by the LEP2 bound up to $M_{A} \approx 500 \mathrm{GeV}$. Even $A \rightarrow h Z$ and $H \rightarrow h h$ would be visible at the current LHC in small portions of the parameter space.

\section{Perspectives for Higgs and New Physics}

The last few years were extremely rich and exciting for particle physics. With the historical discovery of a Higgs boson by the LHC collaborations ATLAS and CMS, crowned by a Nobel prize in fall 2013, and the first probe of its basic properties, they witnessed a giant step in the unraveling of the mechanism that breaks the electroweak symmetry and generates the fundamental particle masses. They promoted the SM as the appropriate theory, up to at least the Fermi energy scale, to describe three of Nature's interactions, the electromagnetic, weak and strong forces. However, it is clear that these few years have also led to some frustration as no signal of physics beyond the SM has emerged from the LHC data. The hope of observing some signs of the new physics models that were put forward to address the hierarchy problem, that is deeply rooted in the Higgs mechanism, with Supersymmetric theories being the most attractive ones, did not materialize.

The Higgs discovery and the non-observation of new particles has nevertheless far reaching consequences for supersymmetric theories and, in particular, for their simplest low energy formulation, the MSSM. The mass of approximately $125 \mathrm{GeV}$ of the observed Higgs boson implies that the scale of SUSY-breaking is rather high, at least $O(\mathrm{TeV})$. This is backed up by the limits on the masses of 
strongly interacting SUSY particles set by the ATLAS and CMS searches, which in most cases exceed the TeV range. This implies that if SUSY is indeed behind the stabilization of the Higgs mass against very high scales that enter via quantum corrections, it is either fine-tuned at the permille level at least or its low energy manifestation is more complicated than expected.

The production and decay rates of the observed Higgs particles, as well as its spin and parity quantum numbers, as measured by ATLAS and CMS with the $\approx 25 \mathrm{fb}^{-1}$ data collected at $\sqrt{s}=7+8$ $\mathrm{TeV}$, indicate that its couplings to fermions and gauge bosons are almost SM-like. In the context of the MSSM, this implies that we are close to the decoupling regime and this particle is the lightest $h$ boson, while the other $H / A / H^{ \pm}$states must be heavier than approximately the Fermi scale. This last feature is also backed up by LHC direct searches of these heavier Higgs states.

This drives up to the question that is now very often asked: what to do next? The answer is, for me, obvious: we are only in the beginning of a new era. Indeed, it was expected since a long time that the probing of the electroweak symmetry breaking mechanism will be at least a two chapters story. The first one is the search and the observation of a Higgs-like particle that will confirm the scenario of the SM and most of its extensions, that is, a spontaneous symmetry breaking by a scalar field that develops a non-zero vev. This long chapter has just been closed by the ATLAS and CMS collaborations with the spectacular observation of a Higgs boson. This observation opens a second and equally important chapter: the precise determination of the Higgs profile and the unraveling of the electroweak symmetry breaking mechanism itself.

A more accurate measurement of the Higgs couplings to fermions and gauge bosons will be mandatory to establish the exact nature of the mechanism and, eventually, to pin down effects of new physics if additional ingredients beyond those of the SM are involved. This is particularly true in weakly interacting theories such as SUSY in which the quantum effects are expected to be small. These measurements could be performed at the upgraded LHC with an energy close to $\sqrt{s}=14 \mathrm{TeV}$, in particular if a very high luminosity, $a$ few $\mathrm{ab}^{-1}$, is achieved [43, 44].

At this upgrade, besides improving the measurements performed so far, rare but important channels such as associated Higgs production with top quarks, $p p \rightarrow t \bar{t} H$, and Higgs decays into $\mu^{+} \mu^{-}$and $Z \gamma$ states could be probed. Above all, a determination of the self-Higgs coupling could be made by searching for double Higgs production e.g. in the gluon fusion channel $g g \rightarrow H H$ [45]; this would be a first step towards the reconstruction of the scalar potential that is responsible of electroweak symmetry breaking. This measurement would be difficult at the LHC even with high-luminosity but a proton collider with an energy $\sqrt{s}=30$ to $100 \mathrm{TeV}$ could do the job [44].

In a less near future, a high-energy lepton collider, which is nowadays discussed in various options (ILC, TLEP, CLIC, $\mu$-collider) would lead to a more accurate probing of the Higgs properties [46], promoting the scalar sector to the very high-precision level of the gauge and fermion sectors achieved by the LEP and SLC colliders in the 1990s [4].

At electron-positron colliders, the process $e^{+} e^{-} \rightarrow H Z$ just looking at the recoiling $Z$ boson allows to measure the Higgs mass, the CP parity and the absolute $H Z Z$ coupling, allowing to derive the total decay width $\Gamma_{H}^{t o t}$. One can then measure precisely, already at $\sqrt{s} \approx 250 \mathrm{GeV}$ where $\sigma\left(e^{+} e^{-} \rightarrow H Z\right)$ is maximal, the absolute Higgs couplings to gauge bosons and light fermions from the decay branching ratios. The important couplings to top quarks and the Higgs self-couplings can measured at the $10 \%$ level in the higher-order processes $e^{+} e^{-} \rightarrow t \bar{t} H$ and $e^{+} e^{-} \rightarrow H H Z$ at energies of at least $500 \mathrm{GeV}$ with a high-luminosity.

Besides the high precision study of the already observed Higgs, one should also continue to search for the heavy states that are predicted by SUSY, not only the superparticles but also the heavier Higgs bosons. The energy upgrade to $\approx 14 \mathrm{TeV}$ (and eventually beyond) and the planed order of magnitude (or more) increase in luminosity will allow to probe much higher mass scales than presently. In fact, 
more generally, one should continue to search for any sign of new physics or new particles, new gauge bosons and fermions, as predicted in most of the SM extensions.

In conclusion, it is not yet time to give up on SUSY and more generally on New Physics but, rather, to work harder to be fully prepared for the more precise and larger data set that will be delivered by the upgraded LHC. It will be soon enough to "philosophize" then as the physics landscape will become more clear.

\section{Acknowledgements}

I would like the thank the organisers for their invitation to the conference and their kind hospitality. This work is supported by the ERC Advanced Grant Higgs@LHC.

\section{References}

[1] ATLAS coll., Phys. Lett. B716 (2012) 1; CMS coll., Phys. Lett. B716 (2012) 30.

[2] F. Englert and R. Brout, Phys. Rev. Lett. 13 (1964) 321; P. Higgs, Phys. Rev. Lett. 13 (1964) 508; P. Higgs, Phys. Lett. 12 (1964) 132; G. Guralnik, C. Hagen, T. Kibble, Phys. Rev. Lett. 13 (1964) 585.

[3] For a review of Higgs physics in the SM, see: A. Djouadi, Phys. Rept. 457 (2008) 1.

[4] K. Olive et al., Particle Data Group, Chin. Phys. C38 (2014) 090001.

[5] B.W. Lee, C. Quigg and H.B. Thacker, Phys. Rev. D16 (1977) 1519.

[6] N. Cabibbo et al., Nucl. Phys. B158 (1979) 295; M. Sher, Phys. Rept. 179 (1989) 273; T. Hambye and K. Riesselmann, Phys. Rev. D55 (1997) 7255; J. Ellis et al., Phys. Lett. B679 (2009) 369.

[7] Updated analyses of the Higgs sector can be found in the slides of the talks given at this conference and at the following Moriond Electroweak conference, March 2015.

[8] G. Degrassi et al., JHEP 1208 (2012) 098; F. Bezrukov et al., JHEP 1210 (2012) 140.

[9] S. Alekhin, A. Djouadi and S. Moch, Phys. Lett. B716 (2012) 214.

[10] M. Drees, R. Godbole, P. Roy, Theory and phenomenology of sparticles, World Sci., 2005.

[11] E. Witten, Nucl. Phys. B188 (1981) 513; R. Barbieri and G. Giudice, Nucl. Phys. B306 (1988) 63; M. Papucci, J. Ruderman and A. Weiler, JHEP 1209 (2012) 035.

[12] A. Djouadi, Phys. Rept. 459 (2008) 1; see also Eur. Phys. J. C74 (2014) 2704 for an update.

[13] S. Heinemeyer, W. Hollik and G. Weiglein, Phys. Rept. 425 (2006) 265; M. Carena and H. Haber, Prog. Part. Nucl.P hys. 50 (2003) 63; B. Allanach et al., JHEP 0409 (2004) 044.

[14] A. Djouadi, J. Kalinowski and M. Spira, Comput. Phys. Commun. 108 (1998) 56; A.Djouadi, M.Muhlleitner and M.Spira, Acta. Phys. Polon. B38 (2007) 635.

[15] J. Baglio and A. Djouadi, JHEP 1103 (2011) 055; see also: JHEP 1010 (2010) 064; J. Baglio, A. Djouadi and R. Godbole, Phys. Lett. B716 (2012) 203.

[16] S. Dittmaier et al., the LHC Higgs cross section working group, arXiv:1101.0593 [hep-ph].

[17] A. Djouadi, Eur. Phys. J. C73 (2013) 2498; A. Djouadi, J. Quevillon and R. Vega-Morales, arXiv:1509.03913 [hep-ph].

[18] S. Dittmaier et al., the LHC Higgs cross section working group: arXiv:1201.3084 [hep-ph].

[19] D. Zeppenfeld et al. Phys. Rev. D62 (2000) 013009; A. Djouadi et al., hep-ph/0002258; M. Dührssen et al., Phys. Rev. D70 (2004) 113009.

[20] A. Djouadi and G. Moreau, Eur. Phys. J. C73 (2013) 2512.

[21] A. Djouadi, L. Maiani, G. Moreau, A. Polosa, J. Quevillon, V. Riquer, Eur. Phys. J. C73 (2013) 2650; JHEP 1506 (2015) 168.

[22] A. Djouadi and A. Lenz, Phys. Lett. B715 (2012) 310; E. Kuflik, Y. Nir and T. Volansky, Phys. Rev.Lett. 110 (2013) 091801; A. Denner et al., Eur.Phys.J. C72 (2012) 1992; A. Djouadi, P. Gam- 
bino, B. Kniehl, Nucl. Phys. B523 (1998) 17; A. Djouadi and P. Gambino, Phys. Rev. Lett. 73 (1994) 2528.

[23] CMS coll., Phys. Rev. D86 (2012) 112003; ATLAS coll., Phys. Rev. Lett. 109 (2012) 032001.

[24] See e.g., N. Kauer and G. Passarino, JHEP 1208 (2012) 116.

[25] ATLAS coll., ATLAS-CONF-2014-042; CMS coll., Phys. Lett. B736 (2014) 64.

[26] A. Djouadi, A. Falkowski, Y. Mambrini and J. Quevillon, Eur. Phys. J. C73 (2013) 2455.

[27] A. Djouadi, O. Lebedev, Y. Mambrini and J. Quevillon, Phys. Lett. B709 (2012) 65.

[28] M. Goodman and E. Witten, Phys. Rev. D31 (1985) 3059; M. Drees and M. Nojiri, Phys. Rev.

D48 (1993) 3483; A. Djouadi and M. Drees, Phys. Lett. B484 (2000) 183.

[29] L. Landau, Dokl. Akad. Nauk Ser. Fiz. 60 (1948) 207; C. Yang, Phys. Rev. 77 (1950) 242.

[30] For a review of the CP Higgs issue, see e.g. S. Kraml (ed.) et al., hep-ph/0608079.

[31] T. Plehn, D. Rainwater and D. Zeppenfeld, Phys. Rev. Lett. 88 (2002) 051801; A. Djouadi, R. Godbole, B. Mellado, K. Mohan, Phys. Lett. B723 (2013) 307.

[32] V. Barger et al., Phys. Rev. D49 (1994) 79; B. Grzadkowski, J. Gunion and X. He, Phys. Rev. Lett. 77 (1996) 5172; J. Gunion and J. Pliszka, Phys. Lett. B444 (1998) 136; P. Bhupal Dev et al., Phys. Rev. Lett. 100 (2008) 051801; J. Ellis, V. Sanz and T. You, Phys. Lett. B726 (2013) 244.

[33] M. Carena et al., Eur. J. Phys. C26 (2003) 601; Eur. Phys. J. C73 (2013) 2552.

[34] A. Djouadi and J. Quevillon, JHEP 1310 (2013) 028.

[35] L. Maiani, A. Polosa, V. Riquer, Phys. Lett. B718 (2012) 465; ibid. B724 (2013) 274.

[36] A. Arbey et al., Phys. Lett. B708 (2012) 162; JHEP 1209 (2012) 107; Phys. Lett. B720 (2013) 153.

[37] A. Djouadi et al. (MSSM working group), hep-ph/9901246.

[38] A. Djouadi, J.L. Kneur and G. Moultaka, Comput. Phys. Commun. 176 (2007) 426; M. Muhlleitner et al., Comput. Phys. Commun. 168 (2005) 46.

[39] L. Hall and Y. Nomura, JHEP 03 (2010) 076; G. Giudice and A. Strumia, Nucl. Phys. B858 (2012) 63; N. Bernal, A. Djouadi, P. Slavich, JHEP 0707 (2007) 016.

[40] See e.g., M. Carena et al., Nucl. Phys. B577 (2000) 88.

[41] A. Djouadi et al., Phys. Lett. B435 (1998) 101; Eur. Phys. J. C1 (1998) 149; Eur. Phys. J. C1 (1998) 163; A. Arvanitaki, G. Villadoro, JHEP 02 (2012) 144; A. Delgado et al., Eur.Phys.J.C73 (2013) 2370.

[42] M. Spira et al., Phys. Lett. B264 (1991) 440; Phys. Lett. B318 (1993) 347; Nucl. Phys. B453 (1995) 17; R. Harlander and W. Kilgore, Phys. Rev. D68 (2003) 013001; R. Harlander, S. Liebler and H. Mantler, Comp. Phys. Comm. 184 (2013) 1605.

[43] ATLAS collaboration, arXiv:1307.7292; CMS collaboration, arXiv:1307.7135.

[44] S. Dawson et al., arXiv:1310.8361 [hep-ex]; N. Arkani-Hamed et al. arXiv:1511.06495 [hepph]; J. Baglio, A. Djouadi and J. Quevillon, arXiv:1511.07853 [hep-ph].

[45] See e.g., J. Baglio et al., JHEP 1304 (2013) 151.

[46] M. Bicer et al., arXiv:1308.6176; H. Baer et al., arXiv:1306.6352; G. Arons et al., arXiv:0709.1893; J. Aguilar-Saavedra, hep-ph/0106315; E. Accomando et al., Phys. Rept. 299 (1998) 1; A. Djouadi, Int. J. Mod. Phys. A10 (1995) 1. 